\title{
The Significance of Cortical Cerebellar Microbleeds and Microinfarcts in Neurodegenerative and Cerebrovascular Diseases
}

\author{
A Post-Mortem 7.0-Tesla Magnetic Resonance Study with Neuropathological Correlates
}

\author{
Jacques L. De Reuck ${ }^{a} \quad$ Vincent Deramecourt ${ }^{a-c, h} \quad$ Florent Auger $^{\mathrm{a}, \mathrm{d}}$ \\ Nicolas Durieux ${ }^{a, d} \quad$ Charlotte Cordonnier ${ }^{a, e}$ David Devos $^{a, f, g} \quad$ Luc Defebvre $^{a, g}$ \\ Caroline Moreau $^{\mathrm{a}, \mathrm{g}}$ Dominique Capparos-Lefebvre ${ }^{\mathrm{i}}$ Florence Pasquier $^{\mathrm{a}, \mathrm{b}}$ \\ Didier Leys ${ }^{a, e} \quad$ Claude-Alain Maurage ${ }^{a, c, h} \quad$ Regis Bordet ${ }^{a, f}$

\footnotetext{
a Université de Lille, INSERM U1171, ${ }^{\mathrm{b}}$ Memory Clinic, 'Pathology Department, ${ }^{\mathrm{d}}$ Imaging Platform, Research Pole,

e Stroke Department, ${ }^{f}$ Pharmacology Department, ${ }^{9}$ Movement Disorder Department, Lille University Hospital, and

hINSERM U837, Lille; 'Centre Hospitalier, Wattrelos, France
}

\begin{abstract}
Key Words
Postmortem 7.0-tesla magnetic resonance imaging •

Cortical cerebellar microbleeds · Cortical cerebellar

microinfarcts · Atherosclerotic cerebrovascular disease .

Cerebral amyloid angiopathy · Neurodegenerative

diseases · Vascular dementia
\end{abstract}

\begin{abstract}
Background: As cortical microbleeds and microinfarcts in neurodegenerative and cerebrovascular diseases have been studied predominantly at the level of the cerebral hemispheres and linked to the presence of cerebral amyloid angiopathy (CAA), we aimed at determining with 7.0-tesla magnetic resonance imaging (MRI) whether the causes and the frequency of cortical cerebellar microbleeds (CCeMBs) and microinfarcts (CCeMls) are the same. Materials and Methods: Hundred and four postmortem brains, composed of 29 with pure Alzheimer's disease (AD), 9 with AD associated to CAA, 10 with frontotemporal lobar degeneration, 9 with amyotrophic lateral sclerosis, 10 with Lewy body dis-
\end{abstract}

ease, 12 with progressive supranuclear palsy, 9 with vascular dementia ( $\mathrm{VaD})$, and 16 controls, were examined. On a horizontal section of a cerebellar hemisphere examined with 7.0-tesla MRI, the number CCeMBs and CCeMls were compared between the different disease groups and the control group. The MRI findings were also compared with the corresponding mean values observed on histological examination of a separate standard horizontal section of a cerebellar hemisphere, used for diagnostic purpose. Results: CCeMBs and CCeMls were only significantly increased in the $\mathrm{VaD}$ group. When comparing the diseased patients with and without CAA mutually and with those with arterial hypertension and severe atherosclerotic cerebrovascular disease, only in the latter an increase of CCeMBs and CCeMls was observed. There was an excellent correlation between the MRI and the neuropathological findings. Conclusions: CCeMBs and CCeMls are mainly due to atherosclerotic cerebrovascular disease and not due to CAA. Their increased presence cannot be included to the Boston diagnostic criteria for CAA.

(c) 2015 S. Karger AG, Basel

\section{KARGER 125}

(c) 2015 S. Karger AG, Base

$1015-9770 / 15 / 0392-0138 \$ 39.50 / 0$

E-Mail karger@karger.com

www.karger.com/ced
Jacques L. De Reuck, MD, PhD

Leopold II laan 96

BE-9000 Ghent (Belgium)

E-Mail dereuck.j@gmail.com 


\section{Introduction}

Magnetic resonance imaging (MRI) has shown a high incidence of cortical microbleeds (CMBs) in patients with small-vessel diseases such as cerebral amyloid angiopathy (CAA) and lipohyalinosis [1-3]. They are also found in asymptomatic persons and in those with arterial hypertension and white matter changes $[4,5]$. CMBs are frequently observed in brains of patients suffering from Alzheimer dementia $(\mathrm{AD})$ associated to CAA $[6,7]$. In the absence of CAA, they are mainly linked to disturbances of the blood-brain barrier associated to the neurodegenerative disease itself and mainly consist of small perivascular bleeds [8]. Although nearly visible on naked-eye examination of postmortem brains, they can easily be detected on 7.0-tesla $\mathrm{T}^{*}$-weighted magnetic resonance imaging (MRI) [9].

Until recently, cortical microinfarcts (CMIs) were considered the invisible lesions in clinical-radiological correlation studies that rely on conventional structural MRI [10]. They are also nearly visible on naked eye examination of postmortem brains and best detected by light-microscopic examination [11]. Recently, in vivo detection of CMIs has been demonstrated with high-resolution 7.0-tesla MRI [12]. They usually occur at the latest stage of CAA or of arteriosclerosis [13] and significantly affect cognition in brain aging $[14,15]$. In our recent postmortem 7.0-tesla MRI study, they were significantly prevalent not only in CAA brains and mainly in patients with vascular dementia, but also to a lesser degree in those with Alzheimer dementia and with Lewy body disease [16].

As the impact of cortical CMBs and CMIs in neurodegenerative and cerebrovascular diseases has mainly been studied at the level of the cerebral hemispheres, this study aims at determining with 7.0-tesla MRI and its neuropathological correlates whether the causes and the frequency of cortical cerebellar microbleeds (CCeMBs) and microinfarcts (CCeMIs) are the same.

\section{Materials and Methods}

\section{Patients and Materials}

Hundred and four patients, who were followed up mainly at the Lille University Hospital or at the Wattrelos Hospital, underwent an autopsy. The final disease diagnosis was made according to the validated neuropathological criteria [17]. The cohorts consisted of 38 patients with Alzheimer's disease (AD), 10 with frontotemporal lobar degeneration (FTLD), 9 with amyotrophic lateral sclerosis (ALS), 10 with Lewy body disease (LBD), 12 with progressive supranuclear palsy (PSP), 9 with vascular dementia (VaD), and 16 controls who had no clinical history of dementia or stroke (C).

Post-Mortem MRI of Cerebellar Vascular Micro-Lesions
The diagnosis of $\mathrm{VaD}$ was made according to recently published neuropathological criteria [13]. In two AD brains and in one LBD brain, the additional severe arteriosclerotic cerebrovascular lesions allowed them being considered as mixed $\mathrm{VaD}$ cases.

Moderate to severe CAA was present in $9 \mathrm{AD}$ brains (ADCAA), compared with $29 \mathrm{AD}$ brains without or with mild CAA, in 5 of the $9 \mathrm{VaD}$ ones, in 1 of the $10 \mathrm{LBD}$ ones, and in none in the other groups.

A previous obtained informed consent of the patients or from the nearest family allowed an autopsy for diagnostic and scientific purposes. The brain tissue samples were acquired from the Lille Neuro-Bank of Lille University, federated to the Centre des Resources Biologiques that acted as an institutional review board.

One fresh cerebral hemisphere was deeply frozen for biochemical examination. The remaining hemisphere, the brainstem, and a cerebellar hemisphere were fixed in formalin for 3 weeks.

The patients with neurodegenerative diseases and $\mathrm{VaD}$ were compared regarding the incidence of CCeMBs and CCeMIs to the control group. The overall group of patients with moderate to severe CAA was also compared with the non-CAA diseased group and with the group with pure and mixed atherosclerotic $\mathrm{VaD}$.

\section{Neuropathological Examination}

The standard procedure for diagnosis of the type of neurodegenerative disease consisted of examining samples from the primary motor cortex, the associated frontal, temporal and parietal cortex, the primary and secondary visual cortex, the cingulate gyrus, the basal nucleus of Meynert, the amygdaloid body, the hippocampus, basal ganglia, mesencephalon, pons, medulla, and cerebellum. Slides from paraffin-embedded sections were immunestained for protein tau, $\beta$-amyloid, $\alpha$-synuclein, prion protein, TDP-43, and ubiquitin.

Similar to the CERAD criteria, brains were classified as CAA, when a majority of $\beta$-amyloid-stained vessels were present in at least three of the four examined samples and as not-CAA, when absent or scarce, in case of a few stained vessels in one or two slides [18].

A quantitative evaluation of the number of CCeMBs and CCeMIs was performed on a standard horizontal section through a cerebellar hemisphere at the level of the dentate nucleus, according to a previously described method [19]. The mean ranking scores in AD, AD-CAA, FTLD, ALS, LBD, PSP, and VaD brains were compared with the controls.

\section{MRI Examination}

From each brain one separate horizontal section of a cerebellar hemisphere at the level of the dentate nucleus was submitted to MRI.

We used a 7.0-T MRI Bruker BioSpin SA with an issuer-receiver cylinder coil of 72-mm inner diameter (Ettlingen, Germany), according to a previously described method [9]. The cerebellar section was placed in a plastic box filled with salt-free water, the size of which did not allow significant tissue movements. Three MRI sequences were used: a positioning sequence, a T2 sequence, and a T2* sequence. $^{*}$

The CCeMBs were detected as focal areas of increased signals on the $\mathrm{T}^{*}$ sequence, whereas CCeMIs were observed as small sharply demarcated zones of increased signals on the T2 sequence without signal loss on the T2*-weighed gradient-echo sequence.

The mean prevalence of CCeMBs and CCeMIs in the different groups of neurodegenerative diseases and $\mathrm{VaD}$ was compared with the control group. 
Table 1. Comparison of median age (interquartile range), gender distribution (\%) and vascular risk factors (\%) between the control and the disease groups

\begin{tabular}{llllllll}
\hline Items & $\begin{array}{l}\mathrm{C} \\
(\mathrm{n}=16)\end{array}$ & $\begin{array}{l}\mathrm{AD} \\
(\mathrm{n}=29)\end{array}$ & $\begin{array}{l}\text { AD-CAA } \\
(\mathrm{n}=9)\end{array}$ & $\begin{array}{l}\text { FTLD } \\
(\mathrm{n}=10)\end{array}$ & $\begin{array}{l}\text { ALS } \\
(\mathrm{n}=9)\end{array}$ & $\begin{array}{l}\text { LBD } \\
(\mathrm{n}=10)\end{array}$ & $\begin{array}{l}\text { PSP } \\
(\mathrm{n}=12)\end{array}$ \\
\hline Age, years (IQR) & $68(50-88)$ & $73(63-88)$ & $68(63-85)$ & $68(62-74)$ & $65(57-76)$ & $82^{*}(75-97)$ & $75(72-82)$ \\
Male, \% & 50 & 55 & 56 & 60 & 56 & 70 & 33 \\
Arterial hypertension & 13 & 10 & 40 & 20 & 20 & 40 & $33-86)$ \\
Diabetes & 13 & 10 & 10 & 10 & 10 & 0 & 70 \\
Hypercholesterolemia & 13 & 20 & 17 & 20 & 10 & 20 & 10 \\
Smoking & 0 & 20 & 17 & 10 & 10 & 10 & 10 \\
Antithrombotic drugs & 19 & $40^{*}$ & 40 & 0 & 10 & 40 & 30 \\
\hline
\end{tabular}

$\mathrm{C}=$ Control; $\mathrm{AD}=$ Alzheimer's disease; $\mathrm{AD}-\mathrm{CAA}=$ Alzheimer's disease with cerebral amyloid angiopathy; FTLD = frontotemporal lobar degeneration; ALS = amyotrophic lateral sclerosis; $\mathrm{LBD}=$ lewy body disease; $\mathrm{PSP}=$ progressive supranuclear palsy; $\mathrm{VaD}=\mathrm{vascu}-$ lar dementia.

${ }^{*} \mathrm{p} \leq 0.05 ;{ }^{* *} \mathrm{p} \leq 0.01 ;{ }^{* * *} \mathrm{p} \leq 0.001$.

The 15 brains with CAA were also compared with those diseased brains without CAA, and separately with 8 non-CAA ones with pure and mixed $\mathrm{VaD}$.

The total number of CCeMBs and CCeMIs of each brain was determined by consensus evaluation by three observers (JDR, FA, and ND), blinded to the neuropathological examination. The inter-rater reliability resulted in an interclass correlation coefficient of 0.81 .

\section{Statistical Analyses}

Univariate comparisons of unpaired groups were performed with the Fisher's exact test for categorical data. The non-parametric Mann-Whitney U test was used to compare continuous variables. The significance level, two-tailed, was set at $\leq 0.05$, for marginally significant, $\leq 0.01$ for significant, and $\leq 0.001$ for highly significant.

\section{Results}

No differences were observed regarding the median age and gender distribution between the control and the disease groups except for the LBD patients, who were somewhat older. Arterial hypertension and the use of antithrombotic agents were significantly more frequent in the $\mathrm{VaD}$ patients compared with the controls, whereas antithrombotic agents were also more used in the $\mathrm{AD}$ patients (table 1).

On neuropathological examination of the cerebellar hemisphere, in addition to the increased mean values of CCeMBs and CCeMIs, more arteriosclerotic leptomeningeal vessels were observed in the $\mathrm{VaD}$ brains (fig. 1). On the separate cerebellar section examined by MRI, only the $\mathrm{VaD}$ brains had significantly more CCeMBs and CCeMIs compared with the controls (fig. 2). The percentage num- ber of $\mathrm{VaD}$ brains with CCeMBs and CCeMIs was also significantly higher compared with the control brains with 78 and $100 \%$ in the former and 31 and $19 \%$ in the latter group, respectively (table 2 ).

The CAA brains had no more CCeMBs and CCeMIs than the overall groups of diseased brains without CAA. Also, when comparing CAA brains with those with pure and mixed $\mathrm{VaD}$ without $\mathrm{CAA}$, the latter group had significantly higher mean values of CCeMBs and CCeMIs (table 3).

\section{Discussion}

This study shows that an increased number of CCeMBs and CCeMIs is not correlated to the presence of CAA, but is mainly found in patients with high vascular risk factors, in particular in those with arterial hypertension and severe arteriosclerotic cerebrovascular disease. Although some groups of neurodegenerative diseases are rather small, we had to make this subdivision as the incidence of CMBs and CMIs in the cerebral hemispheres is quite different between them $[16,17,19]$.

There is an excellent correlation between our findings on neuropathological examination and those on MRI. Although there are some differences in the mean numbers, they are not statistically different with a $p$ value of 0.11 for the CCeMBs and 0.21 for the CCeMIs.

We did not examine subtypes of CCeMIs, as done in a previous study [16] as the cerebellar arterial angioarchitecture is completely different from that in cerebral cortex, with only a single type of cortical arterial branches [20]. 

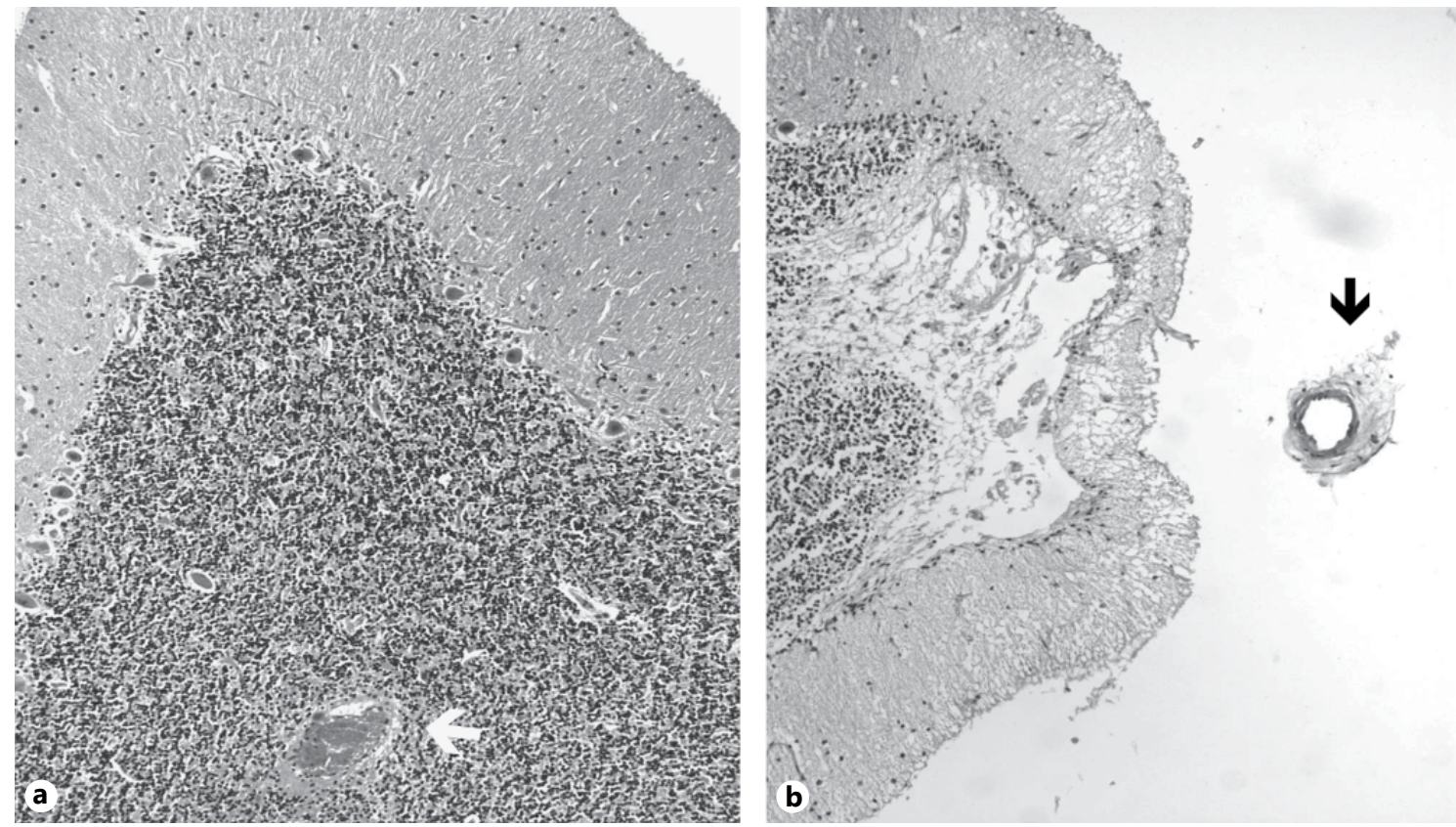

Fig. 1. Histological sections of cerebellar cortex in the brain of a patient with vascular dementia showing a microbleed (arrow) (a) and an old infarct, near a arteriosclerotic leptomeningeal vessel (arrow) (b).
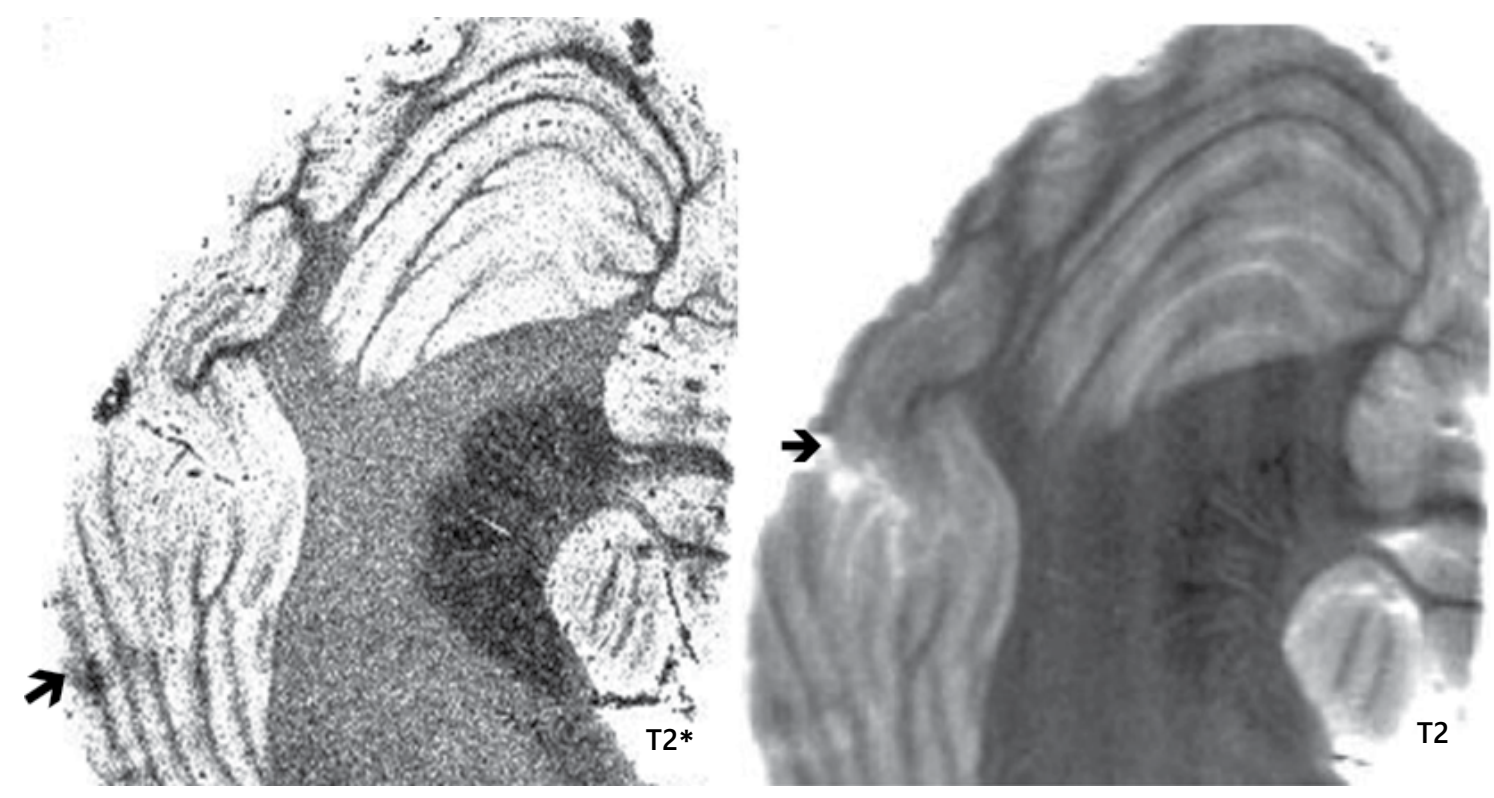

Fig. 2. MRI of a horizontal section of a cerebellar hemisphere in a patient with atherosclerotic vascular dementia. The T2* sequence shows a cortical microbleed (arrow). Note the iron accumulation around the dentate nucleus and in some leptomeningeal postmortem thrombi. T2 MRI of a horizontal section of a cerebellar hemisphere in another patient with atherosclerotic vascular dementia, showing a cortical microinfarct (arrow). 
Table 2. Comparison the mean number (standard deviations) of the cortical cerebellar microbleeds and microinfarcts between the control and disease groups on neuropathological examination and on magnetic resonance imaging

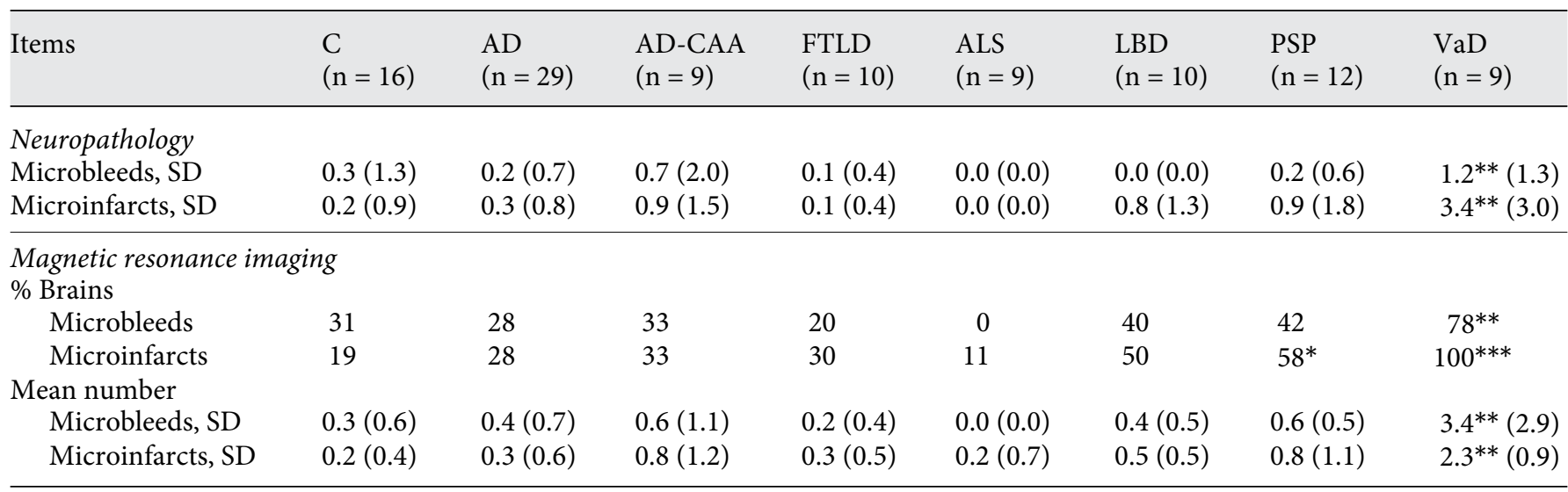

$\mathrm{C}=$ Control; $\mathrm{AD}=$ Alzheimer's disease; $\mathrm{AD}-\mathrm{CAA}=$ Alzheimer's disease with cerebral amyloid Angiopathy; FTLD = frontotemporal lobar degeneration; ALS = amyotrophic lateral sclerosis; LBD = lewy body disease; PSP = progressive supranuclear palsy; VaD = vascular dementia.

${ }^{*} \mathrm{p} \leq 0.05 ;{ }^{* *} \mathrm{p} \leq 0.01 ;{ }^{* * *} \mathrm{p} \leq 0.001$.

Table 3. Mutual comparison on magnetic resonance imaging of the mean number (standard deviations) of cortical cerebellar microbleeds and microinfarcts in the brains of patients with neurodegenerative diseases, without and with cerebral amyloid angiopathy (CAA), and with pure or mixed vascular dementia due to atherosclerotic disease $(\mathrm{AtVaD})$

\begin{tabular}{llll}
\hline Items & Non-CAA $(\mathrm{n}=73)$ & CAA $(\mathrm{n}=15)$ & $\operatorname{AtVaD}(\mathrm{n}=8)$ \\
\hline Microbleeds & $0.7(1.2)$ & $1.0(1.2)$ & $4.5(2.9)^{* *}$ \\
Microinfarcts & $0.9(1.3)$ & $1.4(1.2)$ & $3.0(1.3)^{*}$ \\
\hline
\end{tabular}

${ }^{*} \mathrm{p} \leq 0.05 ;{ }^{* *} \mathrm{p} \leq 0.01$.

In contrast to the high incidence of CMBs and CMIs in the cerebral hemispheres of brains with CAA [16], the latter is not a significant cause of CCeMBs and CCeMIs. The latter are also not increased in the different types of pure neurodegenerative diseases.

Microbleeds are, on the other hand, frequently observed around the dentate nucleus of the cerebellum in AD-CAA and PSP brains, mainly due to the neurodegenerative process itself [17].

A hierachical sequence study of the vascular pathology in $\mathrm{AD}$ showed a difference in progression between CAA and arteriosclerotic disease, the latter affecting more frequently and earlier the cerebellum [21].

CCeMIBs are considered mainly border zone infarcts due to focal cerebellar hypoperfusion either by large vessel occlusive disease or by brain embolism [22]. This is in contrast to small deep microinfarcts and microbleeds, which are more frequently associated to small-artery disease [23].

Isolated cerebellar infarcts may result in subtle cognitive changes that are primarily related to working memory deficit [24] and can contribute to further cognitive impairment in patients with neurodegenerative diseases [25].

In conclusion, CCeMBs and CCeMIs are mainly due to arteriosclerotic cerebrovascular disease and not to CAA. Their increased presence cannot be included in the Boston diagnostic criteria for CAA [26].

\section{Disclosure Statement}

The authors report no conflicts of interest. 


\section{References}

1 Greenberg SM, O’Donnell HC, Schaefer PW, Kraft E: MRI detection of new hemorrhages: potential marker of progression in cerebral amyloid angiopathy. Neurology 1999;53: 1135-1138.

-2 Fazekas F, Kleinert R, Roob G, Kleinert G, Kapeller P, Schmidt R, et al: Histopathologic analysis of foci of signal loss on gradient-echo $\mathrm{T} 2 *$ weighted MR images in patients with spontaneous intracerebral hemorrhage: evidence of microangiopathy-related microbleeds. AJNR Am J Neuroradiol 1999;20:637-642.

-3 Charidimou A, Werring DJ: A raging fire in acute lacunar stroke: inflammation, bloodbrain barrier dysfunction and the origin of cerebral microbleeds. J Neurol Sci 2014;340:12.

$>4$ Roob G, Schmidt R, Kapeller P, Lechner A, Hartung HP, Fazekas F: MRI evidence of past cerebral microbleeds in a healthy elderly population. Neurology 1999;52:991-994.

$>5$ Cordonnier C, Al-Shahi Salman R, Wardlaw J: Spontaneous brain microbleeds: systematic review, subgroup analyses and standards for study design and reporting. Brain 2007;130: 1988-2003.

$\checkmark 6$ De Reuck J, Deramecourt V, Cordonnier C, Leys D, Maurage CA, Pasquier F: The impact of cerebral amyloid angiopathy on the occurrence of cerebrovascular lesions in demented patients with Alzheimer features: a neuropathological study. Eur J Neurol 2011;18: 913-918.

7 De Reuck JL, Cordonnier C, Deramecourt V, Auger F, Durieux N, Bordet R, et al: Microbleeds in postmortem brains of patients with Alzheimer disease: a T2*-weighted gradientecho 7.0 T magnetic resonance imaging study. Alzheimer Dis Assoc Disord 2013;27:162167.

$>8$ De Reuck J: Histopathological stainings and definitions of vascular disruptions in the elderly brain. Exp Gerontol 2012;47:834-837.
9 De Reuck J, Auger F, Cordonnier C, Deramecourt V, Durieux N, Pasquier F, et al: Comparison of 7.0-T T2*-magnetic resonance imaging of cerebral bleeds in post-mortem brain sections of Alzheimer patients with their neuropathological correlates. Cerebrovasc Dis 2011;31:511-517.

10 Smith EE, Schneider JA, Wardlaw JM, Greenberg SM: Cerebral microinfarcts: the invisible lesions. Lancet Neurol 2012;11:272-282.

-11 Costanza A, Xekardaki A, Kovari E, Gold G, Bouras C, Giannakopoulos P: Microvascular burden and Alzheimer-type lesions across the age spectrum. J Alzheimers Dis 2012;32:643652.

12 Van Veluw SJ, Zwanenburg JJ, Engelen-Lee J, Spliet WG, Hendrikse J, Luijten PR, et al: In vivo detection of cerebral cortical microinfarcts with high-resolution 7T MRI. J Cereb Blood Flow Metab 2013;33:322-329.

13 Deramecourt V, Slade JY, Oakley AE, Perry $\mathrm{RH}$, Ince PG, Maurage CA, et al: Staging and natural history of cerebrovascular pathology in dementia. Neurology 2012;78:1043-1050.

14 Kovari E, Gold G, Herrmann FR, Canuto A, Hof PR, Michel JP, et al: Cortical microinfarcts and demyelination significantly affect cognition in brain aging. Stroke 2004;35:410-414.

15 Kalaria RN, Perry RH, O’Brien J, Jaros E: Atheromatous disease in small intracerebral vessels, microinfarcts and dementia. Neuropathol Appl Neurobiol 2012;38:505-508.

$>16$ De Reuck J, Deramecourt V, Auger F, Durieux N, Cordonnier C, Devos D, et al: Postmortem 7.0-tesla magnetic resonance study of cortical microinfarcts in neurodegenerative diseases and vascular dementia with neuropathological correlates. J Neurol Sci 2014; 346:85-89.

17 De Reuck JL: The significance of small cerebral bleeds in neurodegenerative dementia syndromes. Aging Dis 2012;3:307-312.
18 Ellis RJ, Olichney JM, Thal LJ, et al: Cerebral amyloid angiopathy in the brains of patients with Alzheimer's disease: the CERAD experience, PART XV. Neurology 1996;46:15921596.

19 De Reuck J, Deramecourt V, Cordonnier C, Leys D, Pasquier F, Maurage CA: Prevalence of small cerebral bleeds in patients with a neurodegenerative dementia: a neuropathological study. J Neurol Sci 2011;300: 63-66.

20 De Reuck J: The cortico-subcortical arterial angio-architecture in the human brain. Acta Neurol Belg 1972;72:323-329.

21 Thal DR, Ghebremedhin E, Orantes M, Wiestler OD: Vascular pathology in Alzheimer disease: correlation of cerebral amyloid angiopathy and arteriosclerosis/lipohyalinosis with cognitive decline. J Neuropathol Exp Neurol 2003;62:1287-1301.

22 Amarenco P, Kase CS, Rosengart A, Pessin MS, Bousser MG, Caplan LR: Very small (border zone) cerebellar infarcts. Distribution, causes, mechanisms and clinical features. Brain 1993;116:161-186.

23 Park KY, Chung PW, Kim YB, Moon HS, Suh BC, Won YS: Association between small deep cerebellar ischemic lesion and smallvessel disease. Cerebrovasc Dis 2009;28:314320.

24 Hokkanen LS, Kauranen V, Roine RO, Salonen O, Kotila M: Subtle cognitive deficits after cerebellar infarcts. Eur J Neurol 2006;13: 161-170.

25 Saczynski JS, Sigurdsson S, Jonsdottir MK, Eiriksdottir G, Jonsson PV, Garcia ME, et al: Cerebral infarcts and cognitive performance: importance of location and number of infarcts. Stroke 2009;40:677-682.

26 Knudsen KA, Rosand J, Karluk D, Greenberg SM: Clinical diagnosis of cerebral amyloid angiopathy: validation of the Boston criteria. Neurology 2001;56:537-539. 